\title{
SOME INEQUALITIES FOR ENTIRE FUNCTIONS OF EXPONENTIAL TYPE
}

\author{
ROBERT B. GARDNER AND N. K. GOVIL
}

(Communicated by Albert Baernstein II)

ABSTRACT. If $f(z)$ is an asymmetric entire function of exponential type $\tau$,

$$
\|f\|=\sup _{-\infty<x<\infty}|f(x)|,
$$

then according to a well-known result of R. P. Boas,

$$
\left\|f^{\prime}\right\| \leq \frac{\tau}{2}\|f\|
$$

and

$$
|f(x+i y)| \leq \frac{\left(e^{\tau|y|}+1\right)}{2}\|f\|, \quad-\infty<x<\infty,-\infty<y \leq 0 .
$$

Both of these inequalities are sharp. In this paper we generalize the above two inequalities of Boas by proving a sharp inequality which, besides giving as special cases the above two inequalities of Boas, yields some other results as well.

\section{INTRODUCTION AND STATEMENT OF RESULTS}

An entire function is said to be of exponential type $\tau$ if it is of order less than 1 or it is of order 1 and type less than or equal to $\tau$. We will denote this class of functions by $\mathscr{E}_{\tau}$. For $f \in \mathscr{E}_{\tau}$, define $\|f\|=\sup _{-\infty<x<\infty}|f(x)|$. The indicator function $h_{f}(\theta)$ of $f$ is defined by

$$
h_{f}(\theta)=\limsup _{r \rightarrow \infty} \frac{\log \left|f\left(r e^{i \theta}\right)\right|}{r} .
$$

A classical result of Bernstein (see Boas [1, p. 206]) states that if $f \in \mathscr{E}_{\tau}$ and if $\|f\|=1$, then

$$
\left\|f^{\prime}\right\| \leq \tau \text {. }
$$

It is a simple consequence of the Phragmen-Lindelöf principle that (see Boas $[1$, Theorem 6.2.4, p. 82])

$$
|f(x+i y)| \leq e^{\tau|y|}, \quad-\infty<x<\infty,-\infty<y<\infty .
$$

Received by the editors September 7, 1993 and, in revised form, January 27, 1994; the contents of this paper were presented at meeting \#889 of the American Mathematical Society in Cincinnati, Ohio, January 1994.

1991 Mathematics Subject Classification. Primary 30D15; Secondary 30A10, 30E10.

Key words and phrases. Special classes of entire functions and growth estimates, inequalities in the complex domain, approximation in the complex domain.

(C) 1995 American Mathematical Society 
It was proved by Boas [2] that if $h_{f}\left(\frac{\pi}{2}\right)=0$ and $f(x+i y) \neq 0$ for $y>0$, then (1.1) can be replaced by

$$
\left|f^{\prime}(x)\right| \leq \frac{\tau}{2}
$$

and (1.2) by

$$
|f(x+i y)| \leq \frac{\left(e^{\tau|y|}+1\right)}{2}, \quad-\infty<x<\infty,-\infty<y \leq 0 .
$$

For $f \in \mathscr{E}_{\tau}$, we define with respect to a complex number $\zeta$, the function $D_{\zeta}[f]$ as

$$
D_{\zeta}[f]=\tau f(z)+i(1-\zeta) f^{\prime}(z) .
$$

The above definition is due to Rahman and Schmeisser [5].

Note that $\lim _{\zeta \rightarrow \infty} \frac{D_{\zeta}[f(z)]}{\zeta}=-i f^{\prime}(z)$.

In this paper we generalize inequalities (1.3) and (1.4). In fact, as we will see, both these inequalities will turn out to be special cases of our theorem.

We prove

Theorem. Let $f \in \mathscr{E}_{\tau}, h_{f}\left(\frac{\pi}{2}\right)=0,\|f\|=1$ and $f(z)=f(x+i y) \neq 0$ for $\operatorname{Im}(z)>0$. Then

$$
\left|D_{\zeta}[f(z)]\right| \leq \frac{\tau}{2}\left(|\zeta| e^{\tau|y|}+1\right)
$$

for $y=\operatorname{Im}(z) \leq 0$ and $|\zeta| \geq 1$. This result is best possible and the inequality reduces to an equality for $f(z)=\frac{e^{i z z}+1}{2}$ when $\zeta$ is real and $\zeta \geq 1$. [2].

For $\zeta=1$, the above theorem clearly reduces to the inequality (1.4) of Boas

If we divide both sides in the inequality (1.5) of our theorem by $|\zeta|$ and make $|\zeta| \rightarrow \infty$, we get

Corollary 1. Let $f \in \mathscr{C}_{\tau}, h_{f}\left(\frac{\pi}{2}\right)=0,\|f\|=1$ and $f(z) \neq 0$ for $\operatorname{Im}(z)>0$. Then

$$
\left|f^{\prime}(x+i y)\right| \leq \frac{\tau}{2} e^{\tau|y|}, \quad-\infty<x<\infty,-\infty<y \leq 0 .
$$

This result is best possible as is shown by the example given in the theorem.

Taking $y=0$ in our theorem, gives

Corollary 2. Let $f \in \mathscr{E}_{\tau}, h_{f}\left(\frac{\pi}{2}\right)=0,\|f\|=1$ and $f(z) \neq 0$ for $\operatorname{Im}(z)>0$. Then for $|\zeta| \geq 1$,

$$
\left\|D_{\zeta}[f]\right\| \leq \frac{\tau}{2}(|\zeta|+1) .
$$

This result is best possible as is shown by the example given in the theorem.

Corollaries 1 and 2, besides being of independent interest, provide generalizations of inequality (1.3). As is clear, (1.3) is a special case of Corollary 1 when $y=0$, and to obtain (1.3) from Corollary 2, simply divide both sides of (1.7) by $|\zeta|$ and make $|\zeta| \rightarrow \infty$. 


\section{LEMMAS}

For the proof of our theorem, we will need the following lemmas.

Lemma 1. Let $f \in \mathscr{E}_{\tau}$ where $\tau>0$ and $h_{f}\left(\frac{\pi}{2}\right)=0$. If $H$ denotes the (open or closed) upper half-plane, $f(z) \neq 0$ for $z \in H$, then $D_{\zeta}[f(z)] \neq 0$ for $z \in H$ and $|\zeta| \leq 1$.

The above lemma is due to Rahman and Schmeisser [5].

If we apply the above lemma to the function $e^{i \tau z} \overline{f(\bar{z})}$, we will get

Lemma 2. Let $f \in \mathscr{E}_{\tau}$ where $\tau>0$, and let $h_{f}\left(\frac{-\pi}{2}\right)=\tau$. If $L$ denotes the (open or closed) lower half-plane and $f(z) \neq 0$ for $z \in L$, then $D_{\zeta}[f(z)] \neq 0$ for $z \in L$ and $|\zeta| \geq 1$.

Lemma 3. If $f(z)$ is an entire function of exponential type $\tau$ and $|f(x)| \leq M$ for $-\infty<x<\infty$, then

$$
|f(x+i y)| \leq M e^{\tau|y|}, \quad-\infty<x<\infty,-\infty<y<\infty .
$$

This lemma is a simple consequence of the Pragmen-Lindelöf principle (see Govil [3, Lemma 1, p. 226]).

Lemma 4. Let $f \in \mathscr{E}_{\tau}, f(z) \neq 0$ for $\operatorname{Im}(z)>0$, and let $h_{f}(\alpha) \geq h_{f}(-\alpha)$ for some $\alpha, 0<\alpha<\pi$. Then $|f(z)| \geq|f(\bar{z})|$ for $y=\operatorname{Im}(z) \geq 0$.

Lemma 4 is due to Levin [4] (see [1, Theorem 7.8.1]).

Lemma 5. Let $f \in \mathscr{E}_{\tau}, h_{f}\left(\frac{-\pi}{2}\right)=\tau, h_{f}\left(\frac{\pi}{2}\right) \leq 0$, and $f(z) \neq 0$ for $\operatorname{Im}(z) \leq 0$. Then $|f(z)| \geq|g(z)|$ for $\operatorname{Im}(z) \leq 0$ where $g(z)=e^{i \tau z} \overline{f(\bar{z})}$.

Proof. Let $f_{1}(z)=e^{i \tau z / 2} \overline{f(\bar{z})}$. Then $f_{1}(z) \neq 0$ for $\operatorname{Im}(z)>0$. Also, $h_{f_{1}}\left(\frac{-\pi}{2}\right) \leq$ $\frac{\tau}{2}$ and $h_{f_{1}}\left(\frac{\pi}{2}\right)=\frac{\tau}{2}$. Hence by Lemma $4,\left|f_{1}(z)\right| \geq\left|f_{1}(\bar{z})\right|$ for $\operatorname{Im}(z) \geq 0$, which is equivalent to

$$
\left|e^{-i \tau z / 2} f(z)\right| \geq\left|e^{i \tau z / 2} \overline{f(\bar{z})}\right| \text { for } \operatorname{Im}(z) \leq 0,
$$

from which the result follows.

Lemma 6. If $f \in \mathscr{C}_{\tau}$ and $h_{f}\left(\frac{\pi}{2}\right) \leq 0$, then $g \in \mathscr{C}_{\tau}$ where $g(z)=e^{i \tau z} \overline{f(\bar{z})}$.

Proof. Since $f \in \mathscr{E}_{\tau}$ and $h_{f}\left(\frac{\pi}{2}\right) \leq 0$, it is clear that $h_{g}\left( \pm \frac{\pi}{2}\right) \leq \tau$. Hence it follows from a result (see Boas [1, p. 82]) that $h_{g}(\theta) \leq \tau$, which implies that $g \in \mathscr{E}_{\tau}$.

Lemma 7. Let $f \in \mathscr{E}_{\tau}, h_{f}\left(\frac{-\pi}{2}\right)=\tau, h_{f}\left(\frac{\pi}{2}\right) \leq 0$, and let $|f(z)| \geq|g(z)|$ for $\operatorname{Im}(z) \leq 0$, where $g(z)=e^{i \tau z \overline{f(\bar{z})}}$. Then for $|\alpha|>1, h_{g(z)-\alpha f(z)}\left(\frac{-\pi}{2}\right)=\tau$.

Proof. Since $|f(z)| \geq|g(z)|$ for $\operatorname{Im}(z) \leq 0$, we have $|f(-i y)| \geq|g(-i y)|$ for $y \geq 0$. Therefore

$$
|g(-i y)-\alpha f(-i y)| \geq|f(-i y)|\left(|\alpha|-\left|\frac{g(-i y)}{f(-i y)}\right|\right)
$$

for $y \geq 0$ and so

$$
h_{g(z)-\alpha f(z)}\left(\frac{-\pi}{2}\right) \geq \limsup _{y \rightarrow \infty} \frac{\log \left(|f(-i y)|\left\{|\alpha|-\left|\frac{g(-i y)}{f(-i y)}\right|\right\}\right)}{y}=\tau
$$


because $\frac{|g(-i y)|}{|f(-i y)|} \leq 1$ and $|\alpha|>1$. Thus

$$
h_{g(z)-\alpha f(z)}\left(\frac{-\pi}{2}\right) \geq \tau .
$$

On the other hand, the facts that $f \in \mathscr{E}_{\tau}$ and, by Lemma 6, that $g \in \mathscr{E}_{\tau}$ imply that $g(z)-\alpha f(z)$ being a linear combination of $f$ and $g$ is also in $\mathscr{E}_{\tau}$, which implies that

$$
h_{g(z)-\alpha f(z)}\left(\frac{\pi}{2}\right) \leq \tau .
$$

The lemma now follows upon combining (2.3) with (2.4).

Lemma 8. Let $f \in \mathscr{C}_{\tau}$ with $\|f\|=1$ and $h_{f}\left(\frac{\pi}{2}\right)=0$. Then for any $z$ with $\operatorname{Im}(z)=y \leq 0$ and $|\zeta| \geq 1$, we have

$$
\left|D_{\zeta}[f(z)]\right|+\left|D_{\zeta}[g(z)]\right| \leq \tau\left(|\zeta| e^{\tau|y|}+1\right),
$$

where $g(z)=e^{i \tau z} \overline{f(\bar{z})}$.

Proof. Let $F(z)=f(z)-\beta e^{i \tau z}$ where $|\beta|>1$. Then by Lemma 3, $|f(z)| \leq$ $e^{\tau|y|}$ for $y=\operatorname{Im}(z)$ and so $F(z) \neq 0$ in $y=\operatorname{Im}(z) \leq 0$. Also,

$$
|F(-i y)| \geq e^{\tau y}(|\beta|-1)
$$

and therefore

$$
h_{F}\left(\frac{-\pi}{2}\right)=\limsup _{y \rightarrow \infty} \frac{\log |F(-i y)|}{y} \geq \tau \text { by }(2.5)
$$

implying that $h_{F}\left(\frac{-\pi}{2}\right)=\tau$. Further, since $h_{f}\left(\frac{\pi}{2}\right)=0$, it follows from the Phragmen-Lindelöf Principle (see Boas [1, Theorem 6.2.4, p. 82]) that $|f(z)| \leq$ 1 for $\operatorname{Im}(z) \geq 0$. Hence for $y=\operatorname{Im}(z) \geq 0$, we have $|F(i y)| \leq 1+|\beta|$, which implies that $h_{F}\left(\frac{\pi}{2}\right) \leq 0$.

Now, let $G(z)=e^{i \tau z} \overline{F(\bar{z})}$. Then $G(z)=e^{i \tau z} \overline{f(\bar{z})}-\bar{\beta}=g(z)-\bar{\beta}$ where $g(z)=e^{i \tau z} \overline{f(\bar{z})}$. Applying Lemma 5 to $F(z)$ we get $|F(z)| \geq|G(z)|$ for $\operatorname{Im}(z) \leq 0$ and therefore by Lemma 7 when applied to $F(z)$ gives that $h_{G(z)-\alpha F(z)}\left(\frac{-\pi}{2}\right)=\tau$ where $|\alpha|>1$. If we now apply Lemma 2 to the function $G(z)-\alpha F(z)$, which is nonzero for $\operatorname{Im}(z) \leq 0$, we get $D_{\zeta}[G(z)-\alpha F(z)] \neq 0$ for $\operatorname{Im}(z) \leq 0$ and $|\zeta| \geq 1$, which implies

$$
\left|D_{\zeta}[G(z)]\right| \leq\left|D_{\zeta}[F(z)]\right|
$$

for $\operatorname{Im}(z) \leq 0$ and $|\zeta| \geq 1$.

Similarly, if we apply Lemma 2 to the function $F(z)=f(z)-\beta e^{i \tau z},|\beta|>1$, we will get $D_{\zeta}[F(z)] \neq 0$ for $\operatorname{Im}(z) \leq 0$ and $|\zeta| \geq 1$, which implies

$$
\left|D_{\zeta}[f(z)]\right| \leq\left|D_{\zeta}\left[e^{i \tau z}\right]\right|
$$

for $\operatorname{Im}(z) \leq 0$ and $|\zeta| \geq 1$.

Therefore

$$
\begin{aligned}
\left|D_{\zeta}[g(z)]\right|-\tau|\beta| & \leq \mid D_{\zeta}[G(z)] \\
& \leq\left|D_{\zeta}[F(z)]\right| \quad \text { by }(2.6) \\
& =\left|D_{\zeta}\left[f(z)-\beta e^{i \tau z}\right]\right| \\
& =\left|\beta D_{\zeta}\left[e^{i \tau z}\right]\right|-\left|D_{\zeta}[f(z)]\right|
\end{aligned}
$$


by suitable choice of the argument of $\beta$ and by (2.7). We therefore get from (2.8)

$$
\left|D_{\zeta}[f(z)]\right|+\left|D_{\zeta}[g(z)]\right| \leq|\beta|\left(\tau+\left|D_{\zeta}\left[e^{i \tau z}\right]\right|\right)=|\beta|\left(\tau+\tau|\zeta| e^{-\tau y}\right),
$$

for $y=\operatorname{Im}(z) \leq 0$ and $|\zeta| \geq 1$, and this is clearly equivalent to

$$
\left|D_{\zeta}[f(z)]\right|+\left|D_{\zeta}[g(z)]\right| \leq|\beta| \tau\left(1+|\zeta| e^{\tau|y|}\right),
$$

for $y=\operatorname{Im}(z) \leq 0$ and $|\zeta| \geq 1$.

If we now let $|\beta| \rightarrow 1$ in (2.10), the result follows.

\section{PRoof of the theorem}

First, suppose $f$ is precisely of type $\tau$. Since $h_{f}\left(\frac{\pi}{2}\right)=0$ and $\|f\|=1$, we have $h_{f}\left(\frac{-\pi}{2}\right)=\tau$ and so $h_{g}\left(\frac{-\pi}{2}\right)=\tau$ and $h_{g}\left(\frac{\pi}{2}\right)=0$. Applying Lemma 5 to $g(z)$ we get $|g(z)| \geq|f(z)|$ for $\operatorname{Im}(z) \leq 0$. So if $|\beta|>1$, then $f(z)-\beta g(z) \neq$ 0 for $\operatorname{Im}(z) \leq 0$ and hence by Lemma $7, h_{f(z)-\beta g(z)}\left(\frac{-\pi}{2}\right)=\tau$. If we now apply Lemma 2 to the function $f(z)-\beta g(z)$, we get $D_{\zeta}[f(z)-\beta g(z)] \neq 0$ for $\operatorname{Im}(z) \leq 0,|\beta|>1$ and $|\zeta| \geq 1$, which is equivalent to

$$
\left|D_{\zeta}[f(z)]\right| \leq\left|D_{\zeta}[g(z)]\right|,
$$

for $\operatorname{Im}(z) \leq 0$ and $|\zeta| \geq 1$. The inequality (1.5) in our theorem now follows upon combining (3.1) with Lemma 8 .

Since $\frac{\tau}{2}\left(|\zeta| e^{\tau|y|}+1\right)$ is an increasing function of $\tau$, the result trivially holds if $f$ is of type less than $\tau$.

\section{REFERENCES}

1. R. Boas, Entire functions, Academic Press, New York, 1954.

2. Inequalities for asymmetric entire functions, Illinois J. Math. 1 (1957), 94-97.

3. N. Govil, An inequality for functions of exponential type not vanishing in a half-plane, Proc. Amer. Math. Soc. 65 (1977), 225-229.

4. B. Levin, On a special class of entire functions and on related extremal properties of entire functions of finite degree, Izv. Akad. Nauk SSSR. Ser. Mat. 14 (1950), 45-84. (Russian)

5. Q. Rahman and G. Schmeisser, Extension of a theorem of Laguerre to entire functions of exponential type, J. Math. Anal. Appl. 122 (1987), 463-468.

Department of Mathematics, East Tennessee State University, Johnson City, TenNESSEE 37614

E-mail address: gardnerr@etsuarts. east-tenn-st.edu

Department of Mathematics, Auburn University, Auburn, Alabama 36849

E-mail address: govilnk@mail. auburn.edu 